Artigo Original

\title{
Análise do comprometimento de fala de sujeitos portadores de Doença de Parkinson na fase inicial e intermediária
}

\author{
Analysis of speech problems in Parkinson Disease patients in initial and \\ intermediate stages
}

Alcidezio Luiz Sales de Barros ${ }^{1}$, Erideise Gurgel da Costa Silveira², Josian Silva de Medeiros ${ }^{3}$, Maria da Conceição Cavalcante da Silveira Lins ${ }^{4}$, Maria Lúcia Gurgel da Costa5 ${ }^{5}$, Daniela Zwirtes Guerra ${ }^{6}$

\section{RESUMO}

Objetivo: Estudar as alterações de fala do Portador de Doença de Parkinson (DP) nos estágios inicial e intermediário. Para tanto a presente pesquisa classificou o portador de DP através da escala de Webster, analisou as queixas mais freqüentes relacionados à fala; avaliou os distúrbios da articulação de fala dos sujeitos em fase inicial e intermediário da patologia e comparou estas alterações nos diferentes estágios. Método: trata-se de pesquisa experimental, transversal, descritiva, analítica. Foram selecionados para o estudo 10 sujeitos de ambos os gêneros, entre 63 a 78 anos. Foi adotado como critério de inclusão, sujeitos nas duas fases supracitadas e como de exclusão sujeitos sob terapia fonoaudiologica. As queixas relacionadas à fala foram coletadas e analisadas através de uma entrevista semi-estruturada. A articulação da fala foi analisada empiricamente através de diálogos, observações, palpações, exercícios isométricos, isocinéticos e isotônicos. Resultados: A queixa mais freqüente foi rigidez na musculatura dos Órgãos Fono-Articulatórios. Na articulação da fala, encontramos alterações na velocidade e ritmo, devido à rigidez e bracinesia, articulação travada e incoordenação de movimentos, que repercutiram na inteligibilidade de fala. Conclusões: Estes dados apontam a importância do diagnostico fonoaudiológico na DP, no sentido de um melhor conhecimento das alterações de fala nas duas fases, que servirão para melhor direcionamento da terapia fonoaudiológica, que repercutirá em uma maior interação com o social, proporcionando melhoria na qualidade de vida.

\section{Unitermos. Doença de Parkinson; Fala; Transtornos da articulação da fala; Fonoaudiologia.}

Citação: Barros ALS, Silveira EGC, Medeiros JS, Lins MCCS, Costa MLG, Guerra DZ. Análise do comprometimento de fala de sujeitos portadores de Doença de Parkinson na fase inicial e intermediária. Rev Neurocienc 2006; 14(2):023-028.

\section{SUMMARY}

Objective: To study the speech alterations of Parkinson Disease patients the initial and intermediate stages. The present research classified the Parkinson Disease patients by the Webster scale; analyzed the most frequent complaints related to speech; evaluated the disturbance of the speech articulation of the initial and intermediate stages of the disease. Method: this is an experimental, transversal, descriptive, analytical research. We selected 10 patients of both genders, range from 63 to 78 years old. We adopt as criterion of inclusion, patients in two stages of the disease and as of exclusion patients under speech therapy. The complaints related to speech had been collected and analyzed by a half-structured interview. The articulation was analyzed empirically through dialogues, interview, comments, palpation, and isometric, isokinetic and isotonic exercises. Results: The most

Trabalho realizado na Clinica de Fonoaudiologia da Universidade Católica de Pernambuco - UNICAP. Fonte de auxilio: Programa Institucional de Base de Iniciação Científica - PIBIC.

1. Médico Neurologista. Mestre em Fonoaudiologia pela PUC de São Paulo, Doutorando em Neuropsiquiatria pela Universidade Federal de Pernambuco, Prof ${ }^{\circ}$ Adjunto I do departamento de Psicologia do CTCH da UNICAP

2. Médica Doutora em Otorrinolaringologia pela USP, Profa Adjunto I da Graduação em Fonoaudiologia e do Mestrado de Ciências da Linguagem da UNICAP

3. Graduando em Fonoaudiologia pela UNICAP, pesquisador do Programa Institucional de Base de Iniciação Científica da UNICAP

4. Fonoaudióloga. Especialista em Patologias da Linguagem pela UNICAP. Mestre em Fonoaudiologia pela PUC - São Paulo. Profa . Adjunto I da UNICAP

5. Fonoaudióloga pela PUC/SP. Mestre em Distúrbios da Comunicação pela PUC/SP, Doutora em Educação pela USP/SP, Professora Colaboradora do Mestrado de Ciências da Linguagem da UNICAP, Professora Adjunto I do Departamento de Fonoaudiologia da UFPE 6. Fonoaudióloga pela UNICAP, Pós Graduanda em Fonoaudiologia no âmbito da Linguagem pela Universidade da Amazônia - UNAMA 
frequent complaint was rigidity in the speech muscles. In the speech articulation, we found alterations in the speed and rhythm, due to rigidity and bradykinesia, immobilized joint and incoordination of movements that they had presented in the speech intelligibility. Conclusions: This data show the importance of the diagnosis of speech and language of the Parkinson Disease patients, to know the speech alterations in these stages, that will be important to the speech therapy, and will bring a better social interaction and quality of life to these patients.

Keywords: Parkinson Disease; Speech; Articulation disorders; Speech Language and Hearing and Sciences. Citation: Barros ALS, Silveira EGC, Medeiros JS, Lins MCCS, Costa MLG, Guerra DZ. Analysis of speech problems in Parkinson Disease patients in initial and intermediate stages. Rev Neurocienc 2006; 14(2):023-028.

\section{INTRODUÇÃO}

A presente pesquisa buscou investigar, estudar e analisar o comprometimento da fala, nos estágios inicial e intermediário, de sujeitos portadores de Doença de Parkinson (DP). Esta patologia é conceituada como "Uma doença progressiva e crônica do sistema nervoso, envolvendo os gânglios da base e resultando em perturbações no tônus, posturas anormais e movimentos involuntários"1. Esta doença possui alterações primordialmente motoras, e a fala por ser um ato motor sofre repercussões.

Inicialmente descrita por James Parkinson, que a nomeou de Paralisia Agitante foi em 1817, aos 62 anos que Parkinson publicou a monografia "An essay on the shaking palsy", a qual veio a se constituir na primeira descrição bem detalhada da moléstia, que hoje tem como epônimo seu nome"2. O diagnóstico é realizado na terceira idade, é clinico e se expressa como "neurodegenerativa com grande prevalência na população idosa. Estima-se em média uma prevalência de 100 a 150 casos para cada 100 mil pessoas"3. Acomete sujeitos independente de sua composição étnica, econômica ou sócio cultural, com ligeira predominância no sexo masculino 4 . A etiologia da DP é desconhecida, muito embora sabe-se que ocorre uma diminuição na produção da dopamina, que é produzida por neurônios localizados na parte compacta da substância negra e exerce uma função inibidora importante no controle central dos movimentos ${ }^{5}$.

Apesar de permanecer obscura a etiologia do processo degenerativo da substância negra localizada no mesencéfalo, evidências recentes sugerem defeitos no metabolismo dos neurônios desta região que poderiam desencadear o processo degenerativo ${ }^{6}$. Em nosso levantamento bibliográfico não obtivemos informações precisas sobre a etiologia da DP. Sabe-se que o corpúsculo de Lewy se constitui o marcador anátomo patológico, o qual pode ser observado no PET Scan (Tomografia por Emissões de Pósitrons).

Em 1936, Lewy descreveu a presença de inclusões eosinófilas em neurônios nigrais de sujeitos que haviam falecidos com DP. Essas inclusões sabe-se hoje, não são exclusivamente da DP, mas constituem-se no principal marcador anatomopatológico da doença ${ }^{4}$. Dentre os distúrbios clínicos encontrados nos portadores de DP temos: Tremor, rigidez, bradicinesia, Instabilidade postural ${ }^{7}$.

O tremor é mais evidente nas partes distais do membro superior, assemelhando-se a "rolar pílulas" ou "contar dinheiro", apresentando-se de forma rítmica. É o sintoma que mais incomoda o sujeito, pode ser observado na língua, mandíbula, pálpebras, pés e raramente na cabeça, surge quando não estamos desempenhando atividade física constitui-se como um "tremor de repouso"3.

A rigidez ou hipertonia (aumento do tônus muscular) é desencadeada durante o movimento passivo dos membros, apresentando dificuldade na amplitude dos movimentos e articulação, "Ela pode ser a causa de cãibras e dores e pode ser sentida pela extensão passiva dos membros acometidos no nível das articulações" O sujeito apresenta quadro de rigidez e dificuldade de relaxamento muscular, o qual constitui o chamado sinal "roda denteada".

A bradicinesia é a lentidão, pobreza de movimentação, o sujeito apresenta dificuldade para dar início aos movimentos, há falta de controle voluntário, alterações na escrita, não possui amplitude de movimentos no processo de escrever, o qual torna a letra cada vez menor (micrografia), seguida de bloqueios de escrita que são interrupções involuntárias ${ }^{3}$. Ocorre também acúmulo de saliva por dificuldade na deglutição de líquidos.

Associado a bradicinesia ocorre uma falta de controle da marcha é observado nos casos de festinação, o sujeito diminui os passos, e acelera sua marcha inclinando o corpo para frente, procurando o centro de gravidade e normalmente caindo ao solo. A dificuldade em iniciar os movimentos desencadeia quedas para frente ou para traz, o que contribui para uma maior incidência de traumatismos crânio encefálico e conseqüentemente, complicações do ponto de vista de doenças agudas (hematoma subdural, extradural).

$\mathrm{Na}$ fala observamos diminuição da intensidade e dificuldade na articulação o qual, em conjunto com a rigidez, altera os movimentos dos músculos responsáveis pela articulação da fala, o qual leva a uma disartria, conceituada como "dificuldades de expressão oral da linguagem devido a distúrbios do tônus e movimentos dos músculos fonadores, secundários a lesões do sistema nervoso" ". As características mais citadas na literatura fonoaudiológica a respeito da fala enquanto processo articulatório (movimentos dos órgãos como: língua, lábios, dentes, bochechas, mandíbula, palato duro, palato mole e úvula), diz respeito à marcada limitação no âmbito dos movimentos da musculatura envolvida nos mecanismos da fala. Existe diminuição ou aumento da velocidade da fala, redução da inteligibilidade de conversação, redução da habilidade de elevar a língua e fala não inteligível e da 
dificuldade de descrição, movimentos não coordenados da língua durante a fala, redução de movimentos alternados da língua ${ }^{7}$. Há autores que relatam que há repetição, omissão, substituição ou distorção das sílabas fricativas, esta é substituída por explosivas, distorções de vogais e de consoantes manifestadas pela substituição de classes diferentes de fonemas ${ }^{9}$.

Vale salientar que na literatura existem poucos trabalhos acerca das alterações de fala existentes em cada estágio de comprometimento, dentre os existentes citamos Dias \& Limongi; Raming et al ${ }^{10,11}$.

No Brasil, com o aumento significativo da população de idosos é provável que ocorra o aumento de casos de DP. Torna-se latente, portanto, a busca do diagnóstico e tratamento precoce, esperando que os danos causados pela doença tenham evolução mais lenta com a terapia fonoaudiológica, a qual tem como objetivo retardar sua evolução. Os resultados desta pesquisa podem ser transferidos a órgão públicos, privados, ONGS e as instituições que atendem e/ou pesquisam os distúrbios em sujeitos com DP.

Diante do exposto, esta pesquisa se propõe a estudar as alterações de fala nos estágios inicial e intermediário da $D P$, as quais ocasionam uma dificuldade na inter-relação e sociabilidade, que por sua vez é traduzida de forma significativa na qualidade de vida do sujeito.

\section{MATERIAL E MÉTODO}

Foram contactados vinte e seis sujeitos de ambos os gêneros, após a classificação da escala de Webster, a qual avalia aspectos motores, clínicos e atividades da vida diária. Esta escala pontua o grau da incapacidade geral, com valores que variam de 1 a 30 e classifica o sujeito em início da doença (de 1 a 10), incapacidade moderada (11 a 20) e doença grave ou avançada (21 a 30) ${ }^{12}$.

A escala de Webster leva em consideração os seguintes aspectos: bradicinesia de mãos (incluindo escrita manual); rigidez e postura global, balanço dos membros superiores, marcha, tremor de cabeça e mãos, face (expressão e mobilidade), seborréia (presença de oleosidade na face e no couro cabeludo), cuidados pessoais e fala (inteligibilidade, disartrofonia) ${ }^{13}$, destacamos que este último aspecto é o enfoque maior da nossa pesquisa.

Tomamos como parâmetros perceptivos de fala velocidade, hesitação, inflexão, sonoridade, ressonância, inicio de rouquidão, variação de intensidade, monotonia, inteligibilidade e inicio de disartria ${ }^{14-16}$.

Em seguida foi aplicada uma entrevista inicial semiestruturada no intuito de coletar dados quanto à história do participante e às queixas subjetivas em relação ás possíveis alterações da fala, tomamos como parâmetros sujeitos que não possuem Doença de Parkinson ${ }^{15}$.

A articulação da fala foi avaliada através de diálogos, observações, palpações e adaptações de alguns exercícios utilizados na clínica fonoaudiológica para os distúrbios de motricidade oral, exercícios para avaliação isométrica (avalia a tonicidade da musculatura), isocinética (avalia a força da musculatura) e isotônica (avalia mobilidade muscular), foram utilizados de forma sistematizada para verificar o estado das bochechas (simetria, rigidez, mobilidade), lábios (rigidez, curtos, ressecado, tremor de repouso, dificuldade de apreensão, entreabertos em repouso, lateralização, vibração, lentidão e dificuldade de movimento), dentes (adequado ou inadequado), palato duro (profundo e ogival), palato mole e úvula (tenso, rígido e simétrico) e língua (tremor, rigidez, vibração) todos envolvidos na produção da fala dos sujeitos do estudo ${ }^{16,17}$.

Foram selecionados dez voluntários, com idade entre 63 a 78 anos para participar desta pesquisa, desenvolvida na Clinica de Fonoaudiologia Manoel de Freitas Limeira da Universidade Católica de Pernambuco, cujo critério de inclusão foi estar em estágio inicial ou intermediário da DP, o critério de exclusão foi sujeitos em tratamento fonoaudiológico no período da entrevista inicial ou estágio avançado da doença. Foram selecionados cinco participantes em estágio inicial e cinco em estágio intermediário da patologia, de acordo com a escala de pontuação de Webster.

Através de um procedimento experimental, monográfico de forma longitudinal, não cego, no qual se constitui em investigar e analisar os dez sujeitos portadores da DP, com objetivo de se obter generalizações sobre sua natureza. A presente pesquisa foi realizada e embasada sob os princípios do método indutivo ${ }^{18}$

A coleta dos dados supra citados foi concluída em três encontros com duração de uma hora, onde foram utilizados: carta de informação ao participante da pesquisa e termo de compromisso, escala de pontuação de Webster, roteiro de entrevista inicial e avaliação semiestruturado, álbum articulatório, livros, revistas, jornais, luvas cirúrgicas, espátulas, filmadora VHS, fitas VHS, k-7, gravador. Os resultados desta pesquisa estão apresentados em forma de tabelas e texto descritivo. Esta pesquisa foi aprovada pelo Comitê de Ética em Pesquisa da Universidade Católica de Pernambuco com o número do protocolo 040/2004 em 14 de Julho de 2004.

\section{RESULTADOS}

\section{Classificação na escala de Webster}

Dentre os voluntários no estágio inicial observamos graus diversos de incapacidade, que variou de seis a dez pontos, já no estágio intermediário de onze a dezoito pontos, do total do comprometimento dos aspectos motores, clínicos e atividades da vida diária analisados.

No estágio inicial de 100\% dos sujeitos, 40\% apresentou fala clara, sonora, ressonante, fácil de entender. Outros $40 \%$ iniciava uma rouquidão com perda de inflexão e ressonância, no entanto com bom volume e ainda fácil de entender. Em 20\%, observamos rouquidão e fraqueza moderadas; monotonia constante sem variações de altura; hesitação, velocidade, dificuldade para ser compreendida com inicio de disartria. E não 
observamos rouquidão e fraqueza acentuadas, constituindo dificuldade de ser ouvido e compreendido.

No estágio intermediário, $40 \%$ dos sujeitos apresentou inicio de uma rouquidão com perda de inflexão e ressonância, com bom volume e ainda fácil de entender, 40\% apresentou rouquidão e fraqueza moderadas; monotonia constante sem variações de altura; hesitação, velocidade, dificuldade para ser compreendida com inicio de disartria. E 20\% também apresentou rouquidão e fraqueza acentuadas, constituindo dificuldade de ser ouvido e compreendido. Verificamos que os sujeitos não apresentaram fala clara, sonora, ressonante, fácil de entender.

2. Queixas mais freqüentes relacionadas à fala no estágio inicial

No estágio inicial (Tabela 1), 20\% dos sujeitos não apresentou queixa, $80 \%$ relatou rigidez dos músculos da face relacionados à fala, articulação travada, lentidão ou menor velocidade de fala, $20 \%$ se queixou de bloqueios de fala, incoordenação dos movimentos, fala tremula, inteligibilidade de fala alterada. Verificamos que os sujeitos não apresentaram aumento da velocidade de fala

3. Queixas mais freqüentes relacionadas à fala no estágio intermediário

No estágio intermediário (Tabela 2), 100\% da amostra relatou queixas e rigidez dos músculos da face relacionados à fala. E destes, $80 \%$ relatou articulação travada, lentidão ou menor velocidade de fala e inteligibilidade de fala alterada, $60 \%$ relatou fala tremula, $40 \%$ com bloqueios de fala e $20 \%$ relatou aumento da velocidade de fala e incoordenação dos movimentos.

4. Avaliação da articulação de fala no estágio inicial e intermediário

No estágio inicial (Tabela 3) observamos os seguintes aspectos:

Bochechas simétricas em $60 \%$ dos sujeitos, com presença de uma leve rigidez em $20 \%$, a qual não impediu o deslocamento do ar em 100\% da amostra. Lábios apresentaram-se trêmulos em 40\%, rígidos e ressecados em $20 \%$, curtos no lábio superior em $20 \%$ e inferior em $40 \%$, o que não impediu de realizar vibração e lateralização em $100 \%$ da amostra. Eles não apresentaram lentidão e dificuldade de movimentação e de apreensão, mantendo-os sempre fechados quando em repouso. Dentes apresentavam-se adequados em $20 \%$ da amostra e inadequados em $80 \%$. Palato duro profundo em $60 \%$ e ogival em $80 \%$. Palato mole e úvula apresentavam-se simétricos em $80 \%$ e não apresentaram tensão ou rigidez. Língua apresentava-se com tremor em $80 \%$, rigidez em $20 \%$ o que não impediu de realizar a vibração em $60 \%$ da amostra.

Tabela 01 - Queixas no estágio inicial da Doença de Parkinson.

\begin{tabular}{|c|c|c|c|c|c|c|}
\hline Sujeitos & 01 & 02 & 03 & 04 & 05 & Total de queixas no estágio inicial \\
\hline Rigidez dos músculos da face & & $\mathrm{X}$ & $\mathrm{X}$ & $\mathrm{X}$ & $\mathrm{X}$ & 04 \\
\hline Articulação travada & & $\mathrm{X}$ & $\mathrm{X}$ & $\mathrm{X}$ & $x$ & 04 \\
\hline Lentidão / menor velocidade de fala & & $\mathrm{X}$ & $\mathrm{X}$ & $\mathrm{X}$ & $x$ & 04 \\
\hline Aumento da velocidade de fala & & & & & & 00 \\
\hline Bloqueios de fala "jatos de fala" & & & & & $x$ & 01 \\
\hline Incoordenação dos movimentos & & & & & $x$ & 01 \\
\hline Fala tremula & & & $\mathrm{X}$ & & & 01 \\
\hline Inteligibilidade de fala alterada & & $\mathrm{X}$ & & & & 01 \\
\hline Não apresentou queixa & $\mathrm{X}$ & & & & & 01 \\
\hline Total de queixas por sujeitos & 00 & 04 & 04 & 03 & 05 & \\
\hline
\end{tabular}

Tabela 02 - Queixas no estágio intermediário da Doença de Parkinson.

\begin{tabular}{lcccccc}
\hline \multicolumn{1}{c}{ Sujeitos } & $\mathbf{0 6}$ & $\mathbf{0 7}$ & $\mathbf{0 8}$ & $\mathbf{0 9}$ & $\mathbf{1 0}$ & Total de queixas no estágio intermediário \\
\hline Rigidez dos músculos da face & $\mathrm{X}$ & $\mathrm{X}$ & $\mathrm{X}$ & $\mathrm{X}$ & $\mathrm{X}$ & 05 \\
Articulação travada & $\mathrm{X}$ & $\mathrm{X}$ & & $\mathrm{X}$ & $\mathrm{X}$ & 04 \\
Lentidão / menor velocidade de fala & & $\mathrm{X}$ & $\mathrm{X}$ & $\mathrm{X}$ & $\mathrm{X}$ & 04 \\
Aumento da velocidade de fala & $\mathrm{X}$ & & & & & 01 \\
Bloqueios de fala "jatos de fala" & $\mathrm{X}$ & & $\mathrm{X}$ & & & 02 \\
Incoordenação dos movimentos & & $\mathrm{X}$ & & & & 01 \\
Fala tremula & $\mathrm{X}$ & $\mathrm{X}$ & & $\mathrm{X}$ & & 04 \\
Inteligibilidade de fala alterada & & & & & $\mathrm{X}$ & 00 \\
Não apresentou queixa & 06 & 06 & 04 & 04 & 04 & \\
\hline Total de queixas por sujeitos & & & &
\end{tabular}


Tabela 03 - Articulação da fala nos estágios inicial e intermediário da Doença de Parkinson.

\begin{tabular}{|c|c|c|c|}
\hline Estágio inicial & Quantidade de sujeitos & Estágio intermediário & Quantidade de sujeitos \\
\hline Bochechas & & Bochechas & \\
\hline Simétrica & 03 & Simétrica & 04 \\
\hline Rígida & 01 & Rígida & 02 \\
\hline Mobilidade & 05 & Mobilidade & 05 \\
\hline Lábios & & Lábios & \\
\hline Rigidez & 01 & Rigidez & 01 \\
\hline Curtos superiores & 01 & Curtos superiores & 02 \\
\hline Curtos inferiores & 02 & Curtos inferiores & 00 \\
\hline Ressecados & 01 & Ressecados & 03 \\
\hline Trêmulos & 02 & Trêmulos & 02 \\
\hline Dificuldade de apreensão labial & 00 & Dificuldade de apreensão labial & 02 \\
\hline Entre abertos em raposo & 00 & Entre abertos em raposo & 01 \\
\hline Consegue realizar movimento de lateralização & 05 & Consegue realizar movimento de lateralização & 05 \\
\hline Vibração & 05 & Vibração & 04 \\
\hline Lentidão e dificuldade de movimentação & 00 & Lentidão e dificuldade de movimentação & 02 \\
\hline Dentes & & Dentes & \\
\hline Adequados & 01 & Adequados & 00 \\
\hline Palato duro & & Palato duro & \\
\hline $\begin{array}{l}\text { Profundo } \\
\text { Pron }\end{array}$ & 03 & $\begin{array}{l}\text { Profundo } \\
\text { Proun }\end{array}$ & 04 \\
\hline Ogival & 04 & Ogival & 03 \\
\hline Palato mole e úvula & & Palato mole e úvula & \\
\hline Tenso ou rígido & 00 & Tenso ou rígido & 03 \\
\hline Simétrico & 04 & Simétrico & 03 \\
\hline Língua & & Língua & \\
\hline Tremor & 04 & Tremor & 04 \\
\hline Rigidez & 01 & Rigidez & 02 \\
\hline Realiza vibração & 03 & Realiza vibração & 00 \\
\hline
\end{tabular}

No estágio intermediário (Tabela 3) observamos os seguintes aspectos:

Bochechas simétricas em 80\% com presença de rigidez em 40\%, a qual não impediu o deslocamento do ar em 100\% da amostra. Lábios apresentaram-se trêmulos em $40 \%$, rígidos em $20 \%$, ressecados em $60 \%$, curtos no lábio superior em $40 \%$ e inferior 0\%, o que não impediu de realizar lateralização em $100 \%$ no entanto vibração não foi obtida em $20 \%$ da amostra. Eles apresentaram lentidão, dificuldade de apreensão e movimentação em 40\%, mantendo-os entreabertos quando em repouso em $20 \%$. Dentes apresentavam-se inadequados em $100 \%$ da amostra. Palato duro profundo em $80 \%$ e ogival em $60 \%$. Palato mole e úvula apresentavam-se simétricos, tensos e rígidos em 60\%. Língua apresentava-se com tremor em $80 \%$, rigidez em $40 \%$ e não obtivemos vibração em 100\% da amostra.

\section{DISCUSSÃO}

\section{Classificação na escala de Webster}

No estágio inicial dos aspectos clínicos analisados na escala, a fala ocupa a segunda colocação de incidência de comprometimento juntamente com postura, seborréia e cuidados pessoais. Também observamos maior porcentagem de sujeitos com fala clara, sonora, ressonante, fácil de entender, estes sujeitos possuíam de seis a dez pontos na escala.

No estágio intermediário dos aspectos clínicos analisados na escala a fala ocupa a primeira colocação de incidência de comprometimento, juntamente com a bradicinesia das mãos, balanço dos membros superiores e seborréia. Neste estágio observamos uma maior por- centagem de sujeitos que não apresentaram fala clara, sonora, ressonante, fácil de entender, ou seja, um maior comprometimento de inteligibilidade de fala, estes sujeitos possuíam de onze a dezoito pontos na escala.

Estes dados empíricos apontam no sentido de que o comprometimento da fala apresenta uma relação direta com o avanço da patologia, o que sugere a importância da classificação.

Salientamos que na literatura pesquisada, encontramos poucos estudos acerca das alterações existentes em cada estágio de comprometimento, dentre os existentes citamos Dias \& Limongi; Ramig et al ${ }^{10,11}$. Nesta perspectiva este estudo contribui para um melhor conhecimento acerca desta patologia.

\section{Queixas mais freqüentes relacionadas à fala}

No estágio inicial observamos que as queixas mais freqüentes referiram-se a rigidez dos músculos da face relacionados à fala, articulação travada e lentidão ou menor velocidade de fala ${ }^{3,10}$.

No estágio intermediário observamos que 100\% da amostra relata queixas referentes à fala, em especifico a rigidez dos músculos da face. Em seguida, articulação travada, lentidão ou menor velocidade de fala e inteligibilidade de fala alterada esta ultima como pode se visto nas tabelas 1 e 2 , aumenta de uma para quatro queixas o que sugere agravamento na produção e compreensão desta, ou seja, comprometimento de inteligibilidade de fala, o que nos faz pensar em uma correlação entre o avançar dos estágios e o aumento das queixas relacionadas à fala.

Verificamos que a literatura pesquisada refere à dimi- 
nuição ou aumento da velocidade de fala, movimentos não coordenados da língua durante a fala, redução de movimentos alternados da língua, redução da inteligibilidade de conversação, acarretando em uma fala não inteligíve $\left.\right|^{3,7,19}$. No entanto estas fontes bibliográficas não deixam claro em que estágio de comprometimento o sujeito se encontra.

3. Avaliação da articulação de fala estágio inicial e intermediário

Nos sujeitos em estágio inicial verificamos nas bochechas uma simetria em $60 \%$, enquanto que no estágio intermediário observamos $80 \%$, com rigidez bilateral em $40 \%$, divergindo do estágio inicial que apresenta $20 \%$. Verificamos que nos sujeitos em estágio intermediário apesar de apresentarem melhor simetria, boa mobilidade de bochechas e apenas $20 \%$ a mais de rigidez, estes apresentam maior comprometimento de fala.

Observamos nos sujeitos em estágio inicial e intermediário apresentaram tremor nos lábios em 40\% este pode ser encontrado além dos lábios na língua ${ }^{20}$, porém no estágio inicial não observamos lentidão, dificuldade de movimentação e de apreensão, mantendo-os sempre fechados quando em repouso, contrapondo-se aos achados encontrados no estágio intermediário, no qual apresentaram lentidão, dificuldade de apreensão e movimentação em 40\%, mantendo-os entreabertos quando em repouso em $20 \%$.

No estágio inicial os dentes apresentavam-se adequados em $20 \%$ e o restante apresentavam-se inadequados, enquanto que no estágio intermediário apresentavam-se inadequados em $100 \%$ da amostra, destacamos que estes interferem de forma significativa na dinâmica da fala ${ }^{16}$.

No estágio inicial a avaliação das estruturas de palato duro apresentou-se profundo em $60 \%$ e ogival em $80 \%$. Quanto às estruturas de Palato mole e úvula apresentamse simétricas em $80 \%$ e não apresentaram tensão ou rigidez. No estágio intermediário o palato duro apresen- tava-se profundo em $80 \%$ e ogival em $60 \%$. Palato mole e úvula apresentavam-se simétricos, tensos e rígidos em $60 \%$. Verificamos que o palato duro apresentava-se profundo $20 \%$ a mais no estágio intermediário, este estágio se apresentou $20 \%$ menos ogival. Já o palato mole e úvula no estágio inicial apresentam-se simétrico em $20 \%$ a mais que o estágio intermediário, que se apresenta mais tenso e rígido que o estágio intermediário.

Na língua observamos tremor em $80 \%$ dos sujeitos, apresentando-se compatível o estágio inicial e intermediário, entretanto vibração e rigidez divergem entre os estágios, com relação ao aspecto de vibração $40 \%$ no estágio inicial não conseguiram realizar e observamos rigidez em $20 \%$ dos participantes, no estágio intermediário 100\% não conseguiram vibrar a língua e $40 \%$ apresentaram rigidez, a literatura relata redução de movimentos alternados de língua?. Verificamos também que a não realização de vibração de língua apresenta uma relação com a inadequação dos dentes que se apresentou inadequada em $100 \%$ dos sujeitos em estágio intermediário.

\section{CONCLUSÃO}

Concluímos que a classificação dos sujeitos através da escala de Webster em estágios distintos, do conhecimento das queixas mais freqüentes e dos distúrbios da articulação de fala, proporcionou uma melhor compreensão aos profissionais envolvidos no tratamento. Desta maneira propiciará uma terapia direcionada a cada sujeito de uma maneira individualizada, no sentido de se obter um retardo no avanço da doença. Pensamos que este conhecimento propiciará uma melhoria na inter-relação e sociabilidade do sujeito portador desta doença, o que refletirá de uma maneira positiva na sua qualidade de vida. Destacamos a importância do tratamento fonoaudiológico, para um retardo dos sintomas desta patologia. Visto que em nossos resultados observamos um avanço individual, gradual e progressivo da DP.

\section{REFERÊNCIAS BIBLIOGRÁFICAS}

1- O'Sullivan SB. Doença de Parkinson. In: O'Sullivan, Schmitz TJ. Fisioterapia: Avaliação e Tratamento. São Paulo: Manole, 1993, p.549.

2- Barbosa ER, Teive HAG. Doença de Parkinson: Aspectos Históricos. In Andrade LAF, Barbosa ER, Cardoso F, Teive HAG. Doença de Parkinson: estratégias atuais de tratamento. São Paulo: Lemos, 1999, p.13.

3- Teive HAG. Doença de Parkinson: conceitos gerais. Lee Silverman voice treatment (LSVT): tratamento intensivo em pacientes com doença de Parkinson. Doença de Parkinson: Um Guia Prático para Pacientes e Familiares. São Paulo: Lemos, 2000, p. 24-43.

4- Cambier J, Masson M, Dehen H. Manual de Neurologia. Rio de Janeiro: 1999, p.327.

5- Brunner BG. Tratado de enfermagem médico-cirurgico. São Paulo: Guanabara, 2000, p.1512.

6. Doença de Parkinson (endereço na Internet). Brasil. (Última atualização 12/2005; citado em 04/2004). Disponível em: http://parkinson.locaweb.com. $\mathrm{br} /$ doen/default.asp?act $=$ mani.

7- Murdoch BE. Desenvolvimento da fala e distúrbios da linguagem. Rio de Janeiro: Revinter, 1997, p.1; 210.

8- Perelló, J. Transtornos da fala, 5. ed. Rio de Janeiro: Tijuca, 1995, p.02.

9- Metter, EJ. Distúrbios da fala: Avaliação clinica e diagnostico. 1. ed. Rio de janeiro: Enelivros, 1991, p. 152

10. Dias AE, Limongi, JCP. Tratamento dos distúrbios da voz na doença de
Parkinson: o método Lee Silverman. Arq Neuro-Psiquiatr 2003; 61(1):61-66.

11. Raming LO, Countryman S, O'Brien C, Hoehn M. Thompson L. Intensive Speech treatment for patients with Parkinson's disease: short-and long-term comparison of two techniques. Neurology 1996; 47: 1496-1504.

12. Webster DD. Critical analysis of the disability in Parkinson's disease (Endereço na Internet). Espanha. (Última atualização 01/2006; citado em 07/2006). Disponível em: http://www.geocities.com/Heartland/Estates/9451/ eswebste.htm

13. Henderson L, Kennard C, Crawford TJ, Day S, Everitt BS, Goodrich S, et al. Scales for rating motor impairment in Parkinson's disease: stadies of reability an convergent validity. J Neurol Neurosurg Psichiatry 1991; 54: 18-24.

14. Lemos DCH. Disartria. 2. ed. Rio de Janeiro: Enelivros, 1992, p. 19.

15. Metter EJ. Distúrbios da fala: avaliação clinica e diagnóstico. 1. ed. Rio de janeiro: Enelivros, 1991, p. 152.

16. Behlau M, Pontes P. A Avaliação da Voz. In Avaliação e tratamento das disfonias. São Paulo: Lovise, 1995

17. Guimarães I. Protocolo de Avaliação Orofacial, São Paulo, 1.ed; Lovise, 1995 18- Lakatos EM, Marconi MA. Metodologia Científica. São Paulo: Atlas, 1991, p.39-87.

19- Meneses MS, Teive HAG. Doença de Parkinson. Rio de Janeiro: Guanabara Koogan, 2003. p. 228-229.

20- Piccolotto L, Befi-Lopes DM, Limongi SCO (Org.) Tratado de fonoaudiologia São Paulo: Roca, 2004. p.196. 